\title{
Partial protoporphyrinogen oxidase (PPOX) gene deletions, due to different Alu-mediated mechanisms, identified by MLPA analysis in patients with variegate porphyria
}

Michela Barbaro ${ }^{1,2}$, Maire Kotajärvi ${ }^{2,3}$, Pauline Harper ${ }^{2,3,4}$ and Ylva Floderus ${ }^{2,3,4,5^{*}}$

\begin{abstract}
Variegate porphyria (VP) is an autosomal dominantly inherited hepatic porphyria. The genetic defect in the PPOX gene leads to a partial defect of protoporphyrinogen oxidase, the penultimate enzyme of heme biosynthesis. Affected individuals can develop cutaneous symptoms in sun-exposed areas of the skin and/or neuropsychiatric acute attacks. The identification of the genetic defect in VP families is of crucial importance to detect the carrier status which allows counseling to prevent potentially life threatening neurovisceral attacks, usually triggered by factors such as certain drugs, alcohol or fasting.

In a total of 31 Swedish VP families sequence analysis had identified a genetic defect in 26. In the remaining five families an extended genetic investigation was necessary. After the development of a synthetic probe set, MLPA analysis to screen for single exon deletions/duplications was performed.

We describe here, for the first time, two partial deletions within the PPOX gene detected by MLPA analysis. One deletion affects exon 5 and 6 (c.339-197_616+320del1099) and has been identified in four families, most probably after a founder effect. The other extends from exon 5 to exon 9 (c.339-350_987+229del2609) and was found in one family. We show that both deletions are mediated by Alu repeats.

Our findings emphasize the usefulness of MLPA analysis as a complement to PPOX gene sequencing analysis for comprehensive genetic diagnostics in patients with VP.
\end{abstract}

Keywords: Alu-mediated deletions, Protoporphyrinogen oxidase, PPOX, VP, Variegate porphyria, MLPA

\section{Introduction}

Variegate porphyria (VP, OMIM 176200) is an autosomal dominant disease caused by mutations in the protoporphyrinogen oxidase (PPOX) gene, causing a partial defect of the seventh catalytic step of the haem biosynthetic pathway. The enzyme protoporphyrinogen oxidase (PPOX) [EC 1.3.3.4] may become overloaded under conditions with high demand of hepatic haem biosynthesis leading to the accumulation of phototoxic and/or neurotoxic metabolites. Thus VP is a mixed form of cutaneous and acute porphyria. Overt disease is associated with skin photosensitivity

\footnotetext{
* Correspondence: ylva.floderus@karolinska.se

${ }^{2}$ Centre for Inherited Metabolic Diseases, Karolinska University Hospital, Stockholm, Sweden

${ }_{3}^{3}$ Porphyria Centre Sweden, Karolinska University Hospital, Stockholm, Sweden Full list of author information is available at the end of the article
}

and/or neurogenic symptoms as acute attacks of severe abdominal pain, autonomic disturbance, motor neuropathy and more rarely even psychiatric symptoms [1].

The clinical penetrance of VP is about $40 \%[2,3]$ and symptoms almost never manifest before puberty. Skin symptoms are manifest as skin fragility and blistering in sun exposed areas of the body, and are caused by the accumulation of protoporphyrinogen (the substrate of PPOX) in the skin. The skin symptoms are the same as those presented in porphyria cutanea tarda (PCT) and hereditary coproporphyria (HCP). The neurovisceral symptoms are undistinguishable from those for acute intermittent porphyria (AIP) or HCP. Surplus protoporphyrinogen may cause an allosteric inhibition of porphobilinogen deaminase (PBGD, the third enzyme in the haem biosynthetic pathway) leading to accumulation of the neurotoxic metabolites 
5-aminolevulinic acid (ALA) and porphobilinogen (PBG) [4].

During overt disease the diagnosis of VP is based on clinical symptoms and biochemical laboratory investigations. The porphyrin biochemical pattern characteristic for VP is the presence of a plasma fluorescence marker with a maximum peak at $624-627 \mathrm{~nm}$, increased faecal protoporphyrin and inversed coproporphyrin isomer ratio (i.e. copro III isomer $>$ copro I isomer; normal I > III). The urinary porphyrin metabolite pattern may be normal or abnormal with increased excretion of ALA, PBG and coproporphyrin depending on the degree of the metabolic overload of the haem biosynthetic pathway. Erythrocyte porphyrin concentration is usually normal. The activity of the PPOX enzyme, located in the mitochondrial membrane, is measured in lymphocytes or fibroblasts [4]. Further DNA investigations are usually required in order to easily detect asymptomatic/latent relatives [5]. The detection of carriers is of extreme importance and VP patients or latent carriers, as in all acute porphyrias, shall be encouraged to avoid porphyrogenic agents as e.g. certain drugs, alcohol and low carbohydrate diets etc. [6,7].

The PPOX gene is located on chromosome 1q22-23, has a size of $5.5 \mathrm{~kb}$ and contains 13 exons, one non-coding and 12 coding that encode the 477 amino acid PPOX enzyme.

The prevalence of VP in Sweden has been estimated to be 1:100 000 and the mutation pattern in fourteen families has been reported [5]. Before the present study a total of 20 different mutations have been found among the Swedish VP patients (unpublished data). Homozygous or compound heterozygous mutations, with seriously comprised phenotype since early childhood, have been described [8-10] but no such cases have been identified in Sweden.

In five families with well established clinical and biochemical VP diagnosis, the underlying gene defect was not detected by routine DNA sequencing analysis. The aim of this study was to develop a MLPA probe set to screen for possible deletions/duplications affecting the $P P O X$ gene in these families.

\section{Patients and methods}

\section{Patients}

Patients, biochemically confirmed to be affected by VP, in which $P P O X$ routine analysis by PCR and sequencing had failed to identify a disease causing mutation have been selected for this study. The patients included in this study belong to five apparently unrelated Swedish families (Figure 1). The genetic analysis, that includes 845 nucleotides of the promoter region and the non-coding exon 1 of the PPOX gene, as well as the biochemical investigations used to identify patients and latent carriers have been previously described [5].

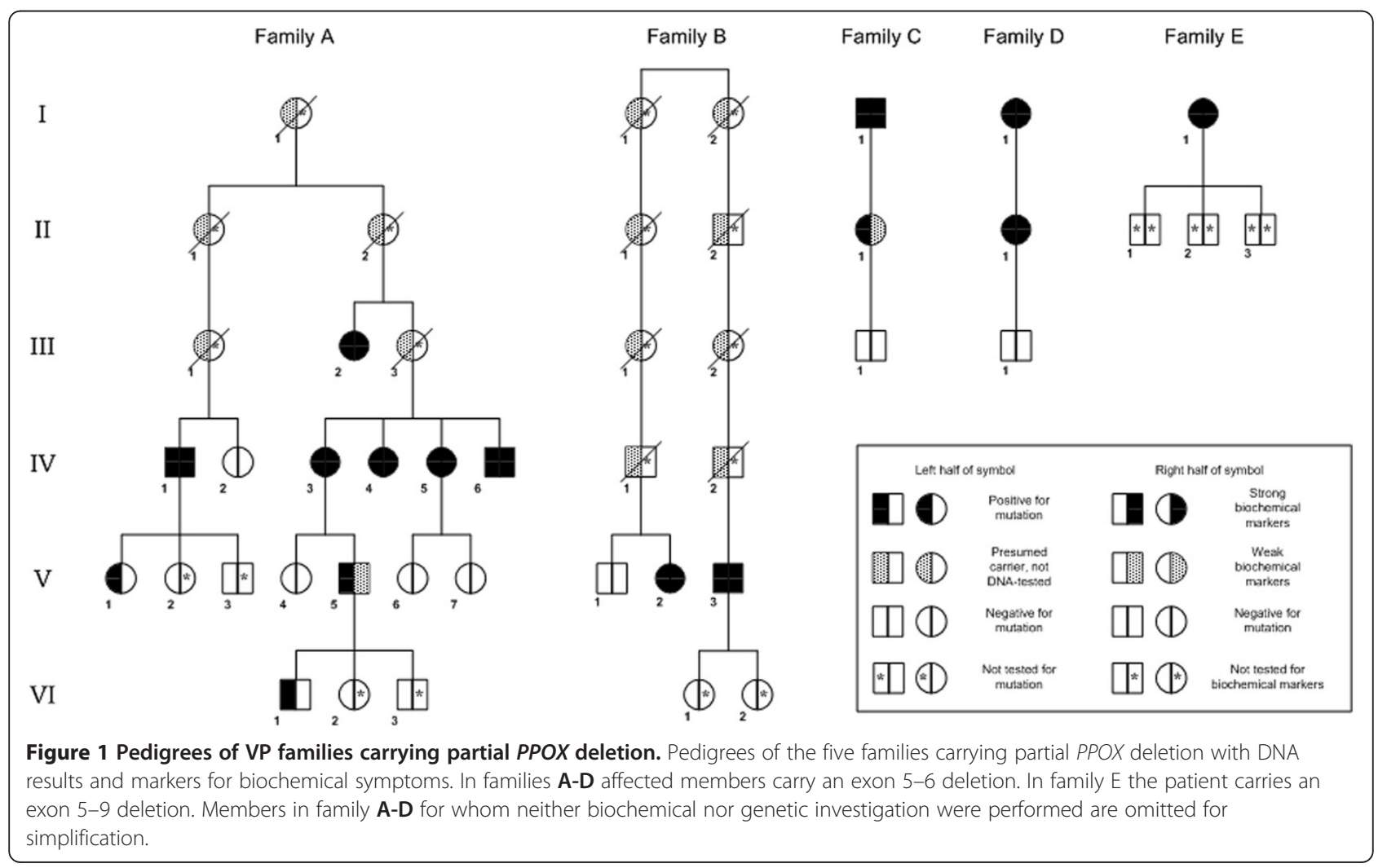


The study was approved by the Ethics Committee of Karolinska Institutet, no. 395/00.

\section{DNA}

Genomic DNA was extracted from peripheral blood samples by the QIAamp DNA Mini kit (QIAGEN, Hilden, Germany), according to the manufacturer's protocol.

\section{MLPA probe set development and analysis}

The target sequence of each synthetic half probe was designed according to the recommendations by Stern et al. [11]. One probe pair for each exon of the PPOX gene was designed, including the first non-coding exon. In the probe set three internal control probe pairs and a pilot probe pair were included [12] as well as a probe targeting the SRY gene on the $\mathrm{Y}$ chromosome to control the correct order of sample loading (see Table 1). MLPA reactions were performed as previously described [12], starting from $100 \mathrm{ng}$ of genomic DNA and using the reagents and the recommendations of the EK1 MLPA reagent kit (MRC-Holland, Amsterdam, The Netherlands). The PCR products were separated by capillary electrophoresis on an ABI 3130XL Genetic Analyzer (Applied Biosystems, Warrington, UK). Trace data were analyzed using the Gene Mapper v4.0 software (Applied Biosystems), and the integrated peak areas and heights were exported to an Excel spreadsheet (Microsoft, Silicon Valley, CA). For each sample, the peak heights were first normalized to the average peak height of the control probes followed by normalization to the average peak height of the control samples included in the run. The sample run was considered acceptable if the ratio for the internal control probe pairs was between 0.8 and 1.2. Threshold values for deletion and duplication were set at 0.75 and 1.25 , respectively.

\section{Deletion breakpoint characterization}

Amplifications of the deletion junctions were performed using a forward primer within intron 3 (5'-TTGG TGGGTCAGATCTTC-3') in combination with a reverse primer within intron 7 (5'-CACTGACAGGTTCATTAC AC-3') for the exon 5-6 deletion or a reverse primer within intron 10 (5'-CCTAAGGAGGGAATATAGC ACTG-3 ${ }^{\prime}$ ) for the exon 5-9 deletion. PCR was carried out in a $50 \mu \mathrm{l}$ reaction mixture containing $100 \mathrm{ng}$ genomic DNA, $0.4 \mu \mathrm{M}$ of each primer, $200 \mu \mathrm{M}$ of each dNTPs, 1X PCR buffer F514L (Finnzyme, Espoo, Finland) and $0.5 \mathrm{U}$ DyNAzyme EXT DNA polymerase (Finnzyme). After an initial denaturing step at $94^{\circ} \mathrm{C}$ for 2 min, 39 cycles, consisting of denaturation at $94^{\circ} \mathrm{C}$ for $30 \mathrm{sec}$, annealing at $57^{\circ} \mathrm{C}$ for $30 \mathrm{sec}$ and elongation at $70^{\circ} \mathrm{C}$ for $1 \mathrm{~min}$, were carried out, followed by a final elongation step at $70^{\circ} \mathrm{C}$ for $10 \mathrm{~min}$. A total of $45 \mu \mathrm{l}$ of PCR products were purified with the Agentcourt $^{\circledR}$ Ampure kit (Beckman Coulter, Brea, CA).
Sequencing reactions of PCR products were performed using the ABI Big Dye Terminator v3.1 kit (Applied Biosystems) followed by ethanol precipitation in a 96-well plate according to manufacturer's protocol, using the same primer as used for the PCR reaction. Sequence reactions were separated by capillary electrophoresis in an ABI 3130XL Genetic Analyzer (Applied Biosystems) and electropherograms were visualized by Sequence Scanner v1.0 (Applied Biosystems).

\section{Haplotyping}

By routine sequencing analysis of the $P P O X$ gene in some members of the four families we determined an intragenic SNP haplotype of the allele carrying the exon 5-6 deletion. The SNPs considered are the validated SNPs from dbSNP [13] within the coding sequence; rs2301286 and rs72714915 within the $5^{\prime}$ UTR and rs2301287 within intron 2 have also been included as data were available from routine analysis and they are quite variable within the Swedish population (Table 2).

\section{Results}

\section{MLPA analysis}

The performance of the PPOX probe set (Table 1) was tested using DNA samples from 11 normal controls. All probes presented a $\mathrm{SD}<10 \%$, thus proving to have a reliable and sufficiently consistent performance.

At least one affected member for each family was analyzed by MLPA. Patient samples belonging to families A, $\mathrm{B}, \mathrm{C}$ and $\mathrm{D}$ showed ratio values consistent with a heterozygous deletion for the probes within exons 5 and 6 (Figure 2). The sample belonging to the patient in family $\mathrm{E}$ showed a deletion extending from exon 5 to exon 9 (Figure 2).

To identify all the carriers of the deletions, all available family members were analyzed by MLPA or PCR; the family trees in Figure 1 summarize the results obtained.

\section{Deletion characterization}

To confirm and fine map the deletions, the deletion junctions were amplified and sequenced. Using a forward primer within intron 3 and a reverse primer within intron 7 , the PCR product in a normal control gave a band of $1904 \mathrm{bp}$, while in the samples with the exons 5-6 deletion a band of approximately $1 \mathrm{~kb}$ was obtained. By sequencing we could also establish that in all four families the affected members share the same deletion of 1099 bp. According to the Human Gene Variation Society [14] the deletion can be described as c.339-197_616+320del1099bp, using the reference sequence NM_000309.3.

In family E the PCR product in the patient had a size of approximately $0.7 \mathrm{~kb}$ instead of the wild type size of $3305 \mathrm{bp}$. By sequencing we determined that the deletion 
Table 1 PPOX probe mix

\begin{tabular}{|c|c|c|c|c|c|c|c|c|}
\hline Probe name & Size $^{a}$ & $5^{\prime}$ half hyb sequence $^{b}$ & $3^{\prime}$ half hyb sequence $^{c}$ & SD & N1 & N2 & Fam A & Fam E \\
\hline GABRA4 $^{d}$ & 84 & CAGCCTGTTGTCATAACCATCG & AGCAAACTGTCCAGGATGCG & 0.20 & nd & nd & nd & nd \\
\hline RELN ${ }^{e}$ & 87 & CAGCATTACGGAATGAAGGTCA & CCACAAGAAGTGGCTTCACAACC & 0.04 & 1.02 & 0.99 & 0.98 & 0.96 \\
\hline PPOXex1 & 90 & GGGAGTAGCGGATTTGAAGCACTT & GTTGGCCTACAGAGGTGTGGCAAG & 0.03 & 0.94 & 1.00 & 1.07 & 1.01 \\
\hline PPOXex7v3 & 93 & CTGAGCGCTGGAGCCAGTGGTCACTT & CGTGGAGGTCTAGAGATGTTGCCTC & 0.04 & 1.00 & 0.99 & 1.03 & 0.55 \\
\hline PPOXex5v5 & 96 & GCAAAGAGCCTGATGAGACTGTGC & ACAGTTTGCCCAGCGCCGCCTTGGACCTG & 0.03 & 1.04 & 1.00 & 0.57 & 0.60 \\
\hline PPOXex6 & 99 & GTGTTGCAGGCAACAGCCGTGAGCTCA & GCATCAGGTCCTGCTITCCCAGTCTCTTC & 0.03 & 1.02 & 1.02 & 0.59 & 0.58 \\
\hline PPOXex3 & 102 & GCTCCGTTCGAGGCCCTAATGGTGCTATCT & TTGAGCTTGGACCTCGGGGAATTAGGCCAG & 0.02 & 1.00 & 0.93 & 1.04 & 1.11 \\
\hline CLDN16 ${ }^{\mathbf{e}}$ & 105 & GACACAAGGGTGTAAAATGCACG & TाTCAGGGTGTGTTTGCATATGATTTAATCAATCAGTATG & 0.04 & 1.00 & 1.02 & 1.01 & 1.00 \\
\hline PPOXex9 & 108 & GTGCCATCACTGCAGTGTCTGTAGCTGTGGTGA & ATCTGCAGTACCAAGGAGCCCATCTGCCTGTCC & 0.03 & 1.05 & 0.99 & 1.02 & 0.58 \\
\hline PPOXex11 & 111 & CTGTTTCAACAGCGGGCCCAGGAAGCAGCTGCTA & CACAATTAGGACTGAAGGAGATGCCGAGCCACTGC & 0.03 & 1.04 & 1.04 & 0.99 & 1.22 \\
\hline PPOXex4 & 114 & CATGCCCTACCCACTGGCCTCAGGTAACACCAGCA & СCTCCGCTCCTTITACTGTGCCCTCATCCTCATATGC & 0.02 & 1.05 & 1.03 & 1.06 & 1.16 \\
\hline PPOXex8 & 117 & CTCATTITCTGGGTCTCTCAAATGTTITCATGCTCTC & AGGTATCTCTAAGGGACAGCAGTCTGGAGGCTGACCAC & 0.04 & 1.06 & 1.03 & 0.98 & 0.56 \\
\hline PPOXex12 & 120 & CTGGCAAAAACTAGGTAAGTTGGGAAAACAGCTGGGCT & GAGGAGGGCCAAGGACATCAGACCCCCAGCTAAAACATTC & 0.03 & 1.06 & 1.09 & 0.99 & 1.16 \\
\hline PPOXex10 & 123 & CTGACGCATGAATGTCCTTCTCTCCAGGGATTTGGACATT & TGGTGCCATCTTCAGAAGATCCAGGAGTCCTGGGAATCGTG & 0.03 & 1.06 & 1.09 & 0.93 & 1.16 \\
\hline PPOXex13 & 126 & GCTGTTAATGACTGTATAGAGAGTGGGCGCCAGGCAGCAGTCA & GTGTCCTGGGCACAGAACCTAACAGCTGATCCCCAACTCTC & 0.03 & 1.08 & 1.03 & 1.04 & 1.13 \\
\hline $\mathrm{RB} 1 \mathrm{e} \times 23^{\mathbf{e}}$ & 129 & GTCACCAATACCTCACATTCCTCGAAGCCCTTACAAGTTTCCT & AGTTCACCCTTACGGATTCCTGGAGGGAACATCTATATTTCACC & 0.02 & 1.00 & 1.02 & 0.98 & 1.11 \\
\hline PPOXex2v4 & 132 & CCTAAGGTGAGTGCTCCACTTGTGCCAGAGGGAGCTTCATTTAATGC & TCTTCCCATTTCCATCAAAAGCTAGATGGATCCTGGCCCTCTG & 0.02 & 1.01 & 1.00 & 1.09 & 1.08 \\
\hline SRY & 135 & CAGTGCAAAGGAAGGAAGAGCTTCTCCGGAGAGCGGGAATATTCT & CTTGCACAGCTGGACTGTAATCATCGCTGTTGAATACGCTTAACATAG & nd & nd & nd & nd & nd \\
\hline
\end{tabular}

${ }^{\mathrm{a}} \mathrm{Size}$ of the ligaton product in bp.

The $5^{\prime}$ half-probes are preceded by the universal tag sequence GGGTCCCTAAGGGTGGA.

'The $3^{\prime}$ half-probes are followed by the universal tag sequence CTAGATTGGATCTTGCTGGCAC and are phosphorylated at the $5^{\prime}$ end.

dPilot probe.

e'Reference probes.

$S D$ standard deviation.
$N d$ not determined. 
Table 2 Haplotypes in the allele with the exon 5-6 deletion

\begin{tabular}{|c|c|c|c|c|c|c|c|c|c|c|}
\hline$\overline{\text { SNP }}$ & SNP alleles & Location & $\begin{array}{c}\text { Average } \\
\text { Heterozygosity }\end{array}$ & MAF & Fam A & Fam B & Fam C & Fam D & $\begin{array}{c}\text { Shared } \\
\text { haplotype }\end{array}$ & $\begin{array}{l}\text { Alleles in Swedish } \\
\text { population }\end{array}$ \\
\hline rs2301286 & $\mathrm{A} / \mathrm{C}$ & Exon 1 UTR & 0.397 & $A=0.3832$ & $\mathrm{C}$ & $\mathrm{C}$ & $\mathrm{C}$ & C & $\mathrm{C}$ & $\mathrm{A} / \mathrm{C}$ \\
\hline rs115158839 & $\mathrm{G} / \mathrm{T}$ & Exon 1 UTR & 0.033 & $T=0.006$ & G & G & G & G & G & G \\
\hline rs72714915 & $C / G$ & Exon 1 UTR & 0.095 & $G=0.0367$ & C & C & C & C & C & $C / G$ \\
\hline rs2301287 & $C / G$ & Intron 2 & 0.444 & $G=0.3759$ & C & C & C & C & C & $C / G$ \\
\hline rs12030747 & $\mathrm{G} / \mathrm{A}$ & Exon 7 (Glu234Lys) & 0.013 & NA & G & G & G & G & G & G \\
\hline rs36013429 & $\mathrm{G} / \mathrm{A}$ & Exon 9 (His304Arg) & 0.008 & $A=0.0476$ & G & G & G & G & G & $G / A$ \\
\hline
\end{tabular}

MAF minor allele frequency.

$N A$ not available.

has a size of $2609 \mathrm{bp}$, thus it can be described as c.339350_987+229del2609bp.

\section{Haplotype analysis}

Patients belonging to the four different families with the exon 5-6 deletion share, on the allele with the deletion, the same haplotype for the 6 SNPs considered (Table 2).

\section{Discussion}

VP is caused by mutations in the PPOX gene. All the 150 mutations reported so far are point mutations or small insertions or deletions [15] with the largest deletion being 23 bp long [16], all of them detectable by a PCR and sequencing approach. In our patient population we had failed to identify a disease causative mutation in five families by

A

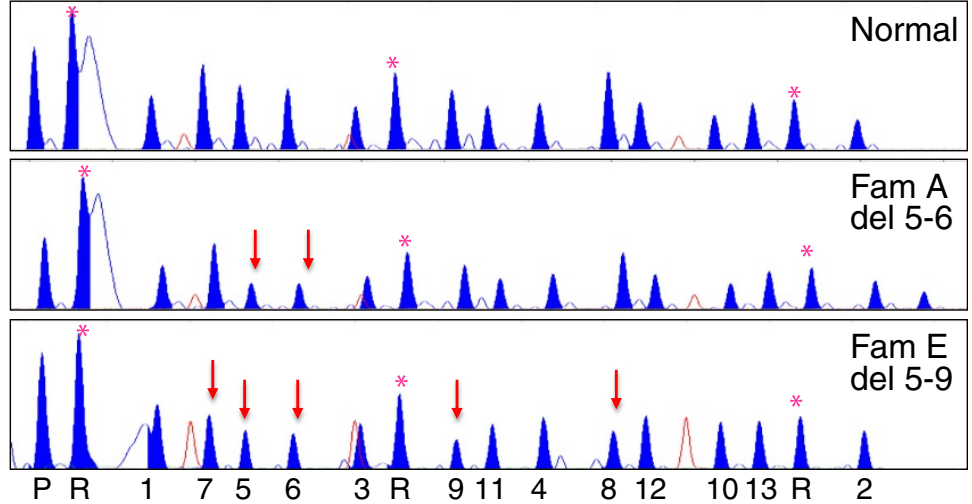

B

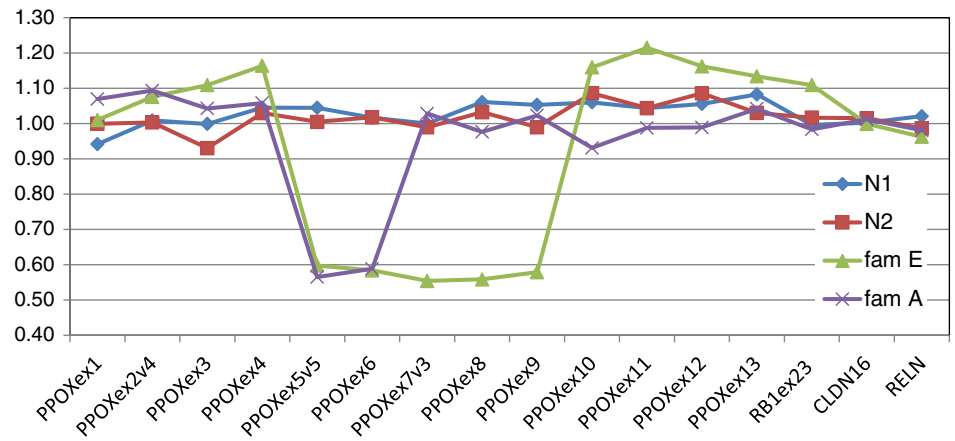

Figure 2 PPOX probe set MLPA results. (A). MLPA trace results for the PPOX probe set in patients belonging to VP families $\mathbf{A}$ and $\mathbf{E}$, and two normal controls (N1 and N2) used as reference. Peaks corresponding to control probes are labeled $\left(^{*}\right)$. On the X-axis at the bottom of the figure the numbers indicate the PPOX exon to which the peak corresponds; $P$ the pilot probe; $R$ the reference probes. Red arrows indicate the deleted probes. (B). Graph showing the analysis results from Excel. Probes on the PPOX gene are ordered according to their location along the gene. Internal reference probes are included and show a ratio value near 1. The ratio values near 0.5 indicate a heterozygous deletion for the corresponding probes. 


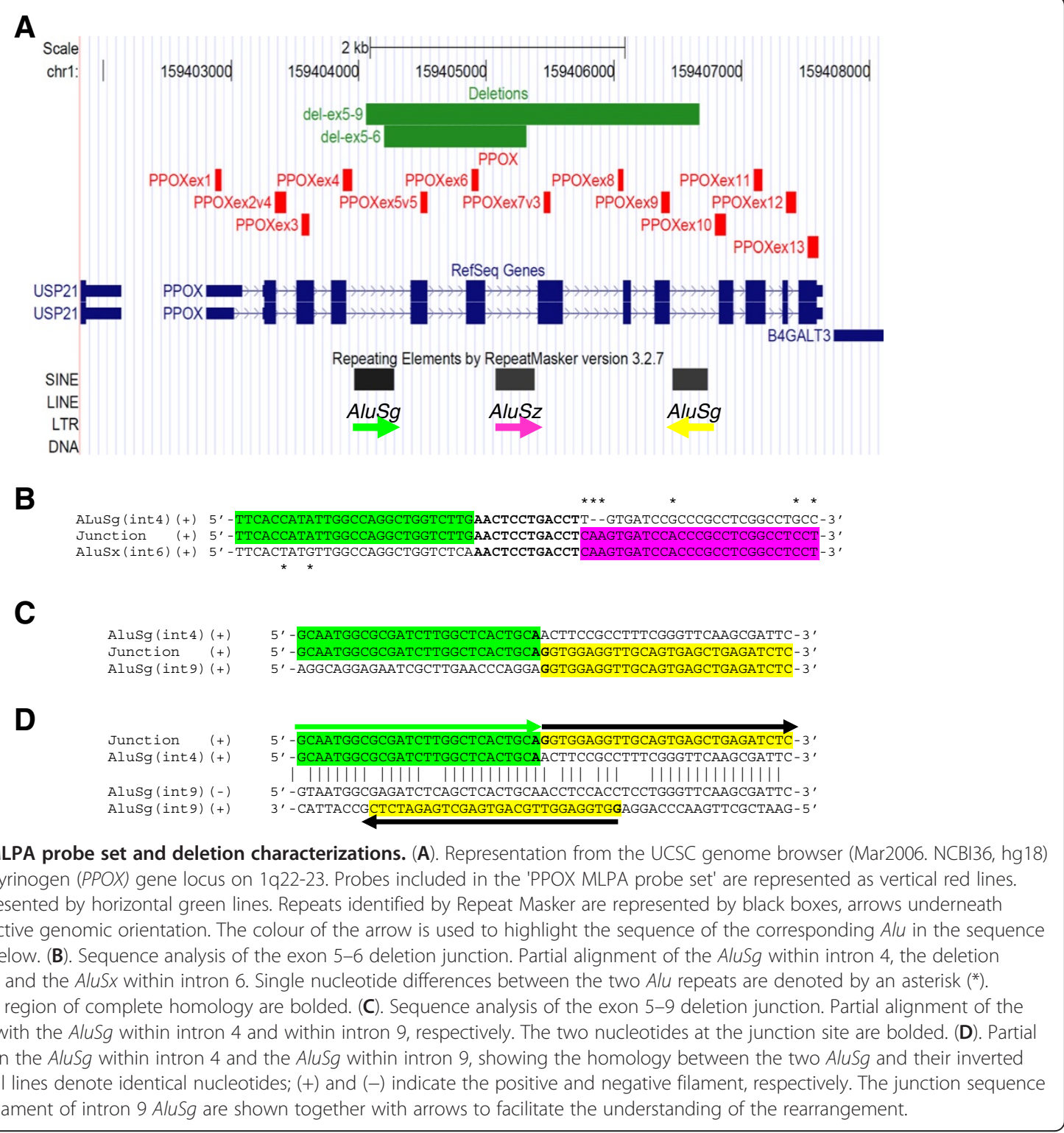

routine sequence analysis. We have therefore developed a MLPA probe set to screen for deletion/duplication of each exon of the PPOX gene that would escape detection by PCR and sequencing.

We identified two deletions, one affecting exon 5 and 6 and the other extending from exon 5 to exon 9. These are the first partial deletions of the PPOX gene described so far.

Both deletions are mediated by Alu sequences. For the exon 5-6 deletion, in silico analysis identified a 308 bp AluSg element within intron 4 and a $353 \mathrm{bp} \mathrm{AluSz}$ element within the intron 6 that share $80 \%$ identity, according to BLASTN alignment [17]. Sequencing of the deletion junction identified the breakpoint within a $12 \mathrm{bp}$ interval of complete homology (Figure 3). As the two Alu elements belong to two different subfamilies and do not share a long stretch of extremely high identity required for homologous recombination, an Alu-mediated non-allelic homologous recombination mechanism (NAHR) is unlikely to be responsible for the deletion. An Alu-specific microhomologymediated deletion is the most likely mechanism [18]. Since we found the same deletion in four independent families we further collected pedigree information and analyzed the gene haplotype to evaluate if this deletion occurred independently due to a recurrent deletion mechanism or if there was a possible founder effect. Patients belonging to the four different families share the same intragenic SNP haplotype on the allele with the exon 5-6 deletion. Furthermore, the oldest family members who are potential carriers of the mutation were born in three neighbouring parishes in the South East of Sweden. Even if we could not find a common 
ancestor between them, it is very likely that they are related, thus suggesting a founder effect for this mutation rather than a recurrent deletion mechanism. This could represent an example of clan genomics: a recently developed mutation of clinical relevance spreading in a specific population [19].

For the exon 5-9 deletion, in silico analysis determined that the 308 bp AluSg element within intron 4 share also $87 \%$ identity with a 278 bp AluSg located within intron 9 with an inverted orientation. Sequencing of the breakpoint region doesn't show a minimal region of homology. It is thus impossible to discriminate if this deletion is mediated by a recombination based mechanism such as NHEJ (non-homologous end joining ) or by a single event of a replication-based mechanism like FoSTeS (Fork Stalling and template switching) or MMBIR (microhomologymediated break-induced replication) $[18,20]$.

Alu-mediated genomic rearrangements have been shown to be involved in $0.3 \%$ of human genetic disease [21] where they can be responsible for both recurrent and nonrecurrent rearrangements [22]. Interestingly the AluSg within intron 9 shares $80 \%$ identity and has inverted orientation not only with respect to the $\mathrm{AluSg}$ within intron 4, where it is involved in the exon 5-9 deletion, but also with respect to the $A l u S z$ within intron 6. As inverted Alu repeats are a threat to genome stability [23], PPOX can be considered at risk for more genetic rearrangements.

Most of the adults positive for the exon 5-6 deletion have presented with biochemical markers indicating VP, mainly typical plasma fluorescence marker and increased faecal porphyrins, with dominance of coproporphyrin III isomer. Clinical symptoms such as skin blisters and/or neuropsychiatric symptoms have been present in several patients and some have previously been misdiagnosed as PCT or AIP, Patient A IV:4 had at her fourth decade of life suffered from severe neuropsychiatric symptoms, abdominal pain, motor neuropathies and periods of hallucinosis. She has later in life developed skin blisters. Patient B V:2 presented with abdominal pain and neurological symptoms at the age of 21 and had an AIP diagnosis at 24. During the fourth decade of life she presented skin symptoms and was diagnosed as PCT. At present she is asymptomatic but has a chronic hypertension and decreased kidney function. Patient D II:1 at the age of 30 had skin lesions resembling PCT and during anaesthesia (caesarean) she developed paralysis of facial muscles. Patient C I:1 was diagnosed at the age of 48 with PCT and later developed as well periods of abdominal pain, depression and mild psychosis. VP diagnosis was lately biochemically confirmed at the age of 77. At that time he had normal excretion of urinary haem precursors but several-fold increased faecal porphyrins and a distinct fluorescence marker in plasma $(627 \mathrm{~nm})$. He died at the age of 88 years. His daughter was identified by genetic analysis at the age of 55 . She has been clinically latent and has weak biochemical markers. Two younger carrier members in Family A (V:1 and VI:1) at the age of 26 and 14 respectively, show neither biochemical signs nor clinical symptoms yet. The patient E I:1 with the exon 5-9 deletion was diagnosed at age 27 after a period of abdominal pain. She has also had temporary skin symptoms. At present she has hypertension and end stage renal disease.

Despite sharing the same mutation these patients present extensive phenotype variation and early diagnosis is of main importance to prevent outbreak of clinical symptoms. Acute porphyria carriers above the age of 50 should be included in yearly liver screening programme including radiological investigation in order to detect primary liver cancer, a known common complication in acute hepatic porphyria [24-27].

Gene dosage analysis has earlier been shown to increase the diagnostic sensitivity for the two other acute porphyrias (AIP and HCP) [28] and large deletions have been reported for the $H M B S$ and $C P O X$ genes, respectively [28-30]. We describe here two partial deletions within the $P P O X$ gene, indicating that partial gene deletions should be considered in the genetic diagnosis of all acute porphyrias. The MLPA probe set we have developed represents a useful tool for the identification of such mutations, and should be performed to complement $P P O X$ sequencing analysis.

\section{Competing interest}

The authors declare that they have no competing interests.

\section{Authors' contributions}

MB has done the experimental planning, developed the MLPA probe kit, analysed and interpreted the genetic results and written the manuscript. MK has assisted in developing the MLPA probe kit, has done the experimental laboratory work and analysed the results. PH has collected the patients, written and evaluated the clinical characterizations. YF has done the experimental planning, collected and evaluated all patient data and written the manuscript. All authors have read and approved the final manuscript.

\section{Author details}

${ }^{1}$ Department of Molecular Medicine and Surgery, Karolinska Institutet, Stockholm, Sweden. ${ }^{2}$ Centre for Inherited Metabolic Diseases, Karolinska University Hospital, Stockholm, Sweden. ${ }^{3}$ Porphyria Centre Sweden, Karolinska University Hospital, Stockholm, Sweden. ${ }^{4}$ Department of Laboratory Medicine, Division of Metabolic Diseases, Karolinska Institutet, Stockholm, Sweden. ${ }^{5}$ CMMS L7:05, Karolinska University Hospital, SE-171 76, Stockholm, Sweden.

Received: 28 August 2012 Accepted: 9 January 2013 Published: 16 January 2013

\section{References}

1. Puy H, Gouya L, Deybach JC: Porphyrias. Lancet 2010, 375:924-937.

2. Hift RJ, Meissner D, Meissner PN: A systematic study of the clinical and biochemical expression of variegate porphyria in a large South African family. Br J Dermatol 2004, 151:465-471.

3. Von Und Zu Fraunberg M, Timonen K, Mustajoki P, Kauppinen R: Clinical and biochemical characteristics and genotype-phenotype correlation in Finnish variegate porphyria patients. Eur J Hum Genet 2002, 10:649-657.

4. Meissner $P$, Adams $P$, Kirsch R: Allosteric inhibition of human lymphoblast and purified porphobilinogen deaminase by protoporphyrinogen and coproporphyrinogen. A possible mechanism for the acute attack of variegate porphyria. J Clin Invest 1993, 91:1436-1444. 
5. Wiman $\AA$, Harper P, Floderus Y: Nine novel mutations in the protoporphyrinogen oxidase gene in Swedish families with variegate porphyria. Clin Genet 2003, 64:122-130.

6. Thunell S, Pomp E, Brun A: Guide to drug porphyrogenicity prediction and drug prescription in the acute porphyrias. Br J Clin Pharmacol 2007, 64:668-679.

7. The Drug Database for Acute Porphyria. http://www.drugs-porphyria.org.

8. Frank J, McGrath J, Lam H, Graham RM, Hawk JL, Christiano AM: Homozygous variegate porphyria: identification of mutations on both alleles of the protoporphyrinogen oxidase gene in a severely affected proband. J Invest Dermatol 1998, 110:452-455

9. Corrigall AV, Hift RJ, Davids LM, Hancock V, Meissner D, Kirsch RE, Meissner PN: Homozygous variegate porphyria in South Africa: genotypic analysis in two cases. Mol Genet Metab 2000, 69:323-330.

10. Kauppinen $R$, Timonen $K$, Von Und Zu Fraunberg M, Laitinen E, Ahola H, Tenhunen R, Taketani S, Mustajoki P: Homozygous variegate porphyria: 20 y follow-up and characterization of molecular defect. $J$ Invest Dermatol 2001, 116:610-613.

11. Stern RF, Roberts RG, Mann K, Yau SC, Berg J, Ogilvie CM: Multiplex ligation-dependent probe amplification using a completely synthetic probe set. Biotechniques 2004, 37:399-405.

12. Barbaro M, Cicognani A, Balsamo A, Lofgren A, Baldazzi L, Wedell A, Oscarson M: Gene dosage imbalances in patients with $46, \mathrm{XY}$ gonadal DSD detected by an in-house-designed synthetic probe set for multiplex ligation-dependent probe amplification analysis. Clin Genet 2008, 73:453-464.

13. The SNP Database. http://www.ncbi.n/m.nih.gov/SNP/.

14. den Dunnen JT, Antonarakis SE: Mutation nomenclature extensions and suggestions to describe complex mutations: a discussion. Hum Mutat 2000, 15:7-12.

15. The Human Gene Mutation Database. http://www.hgmd.cf.ac.uk.

16. Whatley SD, Puy H, Morgan RR, Robreau AM, Roberts AG, Nordmann Y, Elder GH, Deybach JC: Variegate porphyria in Western Europe: identification of PPOX gene mutations in 104 families, extent of allelic heterogeneity, and absence of correlation between phenotype and type of mutation. Am J Hum Genet 1999, 65:984-994.

17. Zhang Z, Schwartz S, Wagner L, Miller W: A greedy algorithm for aligning DNA sequences. J Comput Biol 2000, 7:203-214.

18. Zhang F, Carvalho CM, Lupski JR: Complex human chromosomal and genomic rearrangements. Trends Genet 2009, 25:298-307.

19. Lupski JR, Belmont JW, Boerwinkle E, Gibbs RA: Clan genomics and the complex architecture of human disease. Cell 2011, 147:32-43.

20. Zhang F, Khajavi M, Connolly AM, Towne CF, Batish SD, Lupski JR: The DNA replication FoSTeS/MMBIR mechanism can generate genomic, genic and exonic complex rearrangements in humans. Nat Genet 2009, 41:849-853.

21. Deininger PL, Batzer MA: Alu repeats and human disease. Mol Genet Metab 1999, 67:183-193.

22. Shaw CJ, Lupski JR: Non-recurrent $17 \mathrm{p} 11.2$ deletions are generated by homologous and non-homologous mechanisms. Hum Genet 2005, 116:1-7.

23. Stenger JE, Lobachev KS, Gordenin D, Darden TA, Jurka J, Resnick MA: Biased distribution of inverted and direct Alus in the human genome: implications for insertion, exclusion, and genome stability. Genome Res 2001, 11:12-27.

24. Kauppinen R, Mustajoki P: Acute hepatic porphyria and hepatocellular carcinoma. Brit J Cancer 1988, 57:117-120.

25. Andant C, Puy H, Bogard C, Faivre J, Soulé JC, Nordmann Y, Deybach JC: Hepatocellular carcinoma in patients with acute hepatic porphyria: frequency of occurrence and related factors. J Hepatol 2000, 32:933-939.

26. Schneider-Yin X, van Tuyll van Serooskerken AM, Tyblewski W, PobleteGutierrez P. Minder El, Frank J: Hepatocellular carcinoma in variegate porphyria: a serious complication. Acta Derm Venereol 2010, 90:512-515.

27. Innala $E$, Andersson C: Screening for hepatocellular carcinoma in acute intermittent porphyria: a 15-year follow-up in northern Sweden. J Intern Med 2011, 269:538-545.

28. Whatley SD, Mason NG, Woolf JR, Newcombe RG, Elder GH, Badminton MN: Diagnostic strategies for autosomal dominant acute porphyrias: retrospective analysis of 467 unrelated patients referred for mutational analysis of the HMBS, CPOX, or PPOX gene. Clin Chem 2009, 55:1406-1414.
29. Di Pierro E, Besana V, Moriondo V, Brancaleoni V, Tavazzi D, Casalgrandi G, Ventura P, Rocchi E, Cappellini MD: A large deletion on chromosome 11 in acute intermittent porphyria. Blood Cells Mol Dis 2006, 37:50-54.

30. Barbaro $M$, Kotajärvi $M$, Harper $P$, Floderus $Y$ : Identification of an AluY-mediated deletion of exon 5 in the CPOX gene by MLPA analysis in patients with hereditary coproporphyria. Clin Genet 2011, 81:249-256.

doi:10.1186/1750-1172-8-13

Cite this article as: Barbaro et al:: Partial protoporphyrinogen oxidase (PPOX) gene deletions, due to different Alu-mediated mechanisms, identified by MLPA analysis in patients with variegate porphyria. Orphanet Journal of Rare Diseases 2013 8:13.

\section{Submit your next manuscript to BioMed Central and take full advantage of:}

- Convenient online submission

- Thorough peer review

- No space constraints or color figure charges

- Immediate publication on acceptance

- Inclusion in PubMed, CAS, Scopus and Google Scholar

- Research which is freely available for redistribution 